\title{
Biología, Lenguaje y Evolución: Eric Lenneberg. Fundamentos biológicos del lenguaje [FBL] (1967-2017)
}

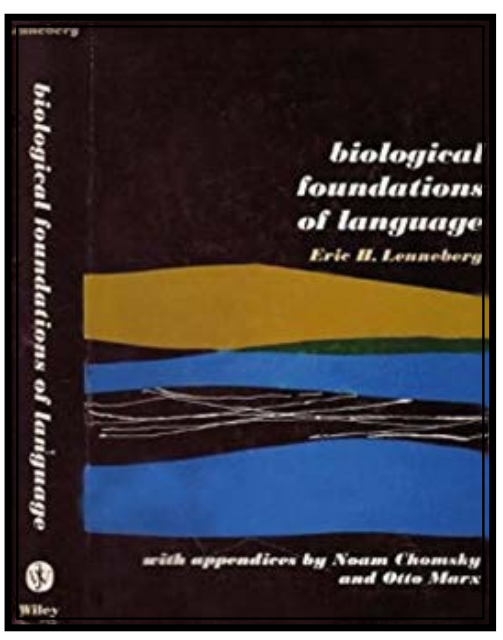

Figura 1. Portada de la edición en inglés 1967

Eric Lenneberg publica en 1967 un libro que se convertiría, con el paso del tiempo, en uno de los primeros tratados consagrados a la biología del lenguaje y a los temas más sugerentes como el lenguaje y la evolución, el cambio lingüístico como correlato de la evolución biológica, el origen 'genético' de las lenguas, el darwinismo lingüístico entre otros, y que le dieron paso a un nuevo campo denominado 'biolingüística' [también biolinguistic enterprise o biolinguistic approach]. Tanto en los Estados Unidos como en Japón y en Europa, diversos autores se han ocupado en estudiar, discutir y replantear las tesis de Lenneberg, entre ellos Noam Chomsky, y es precisamente en el marco de la gramática generativa que se han ido estudiando y proyectando los aportes de Lenneberg. En una reciente entrevista el profesor Chomsky (Chomsky, 2017), elogia el libro de Lenneberg en los siguientes términos: The book developed a sound, deeply informed, comprehensive, carefully executed biological basis for the study of the human language faculty, its use and its evolution, not only bringing together what was known about this topic but substantially extending it, including provocative speculations that were far from conventional at the time but have since in many cases been shown to have been on the right track. And while a great

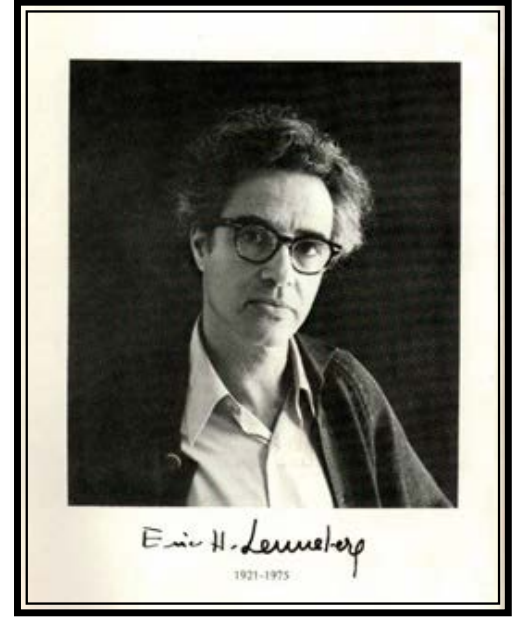

Figura 2. Fotografía de Eric Lenneberg

deal has been learned since, this study remains an indispensable source for inquiry into the biology of language in all of its aspects.

El libro 'Fundamentos biológicos del lenguaje' es el resultado de una sólida formación en psicología, biología y lingüística por parte de Lenneberg. Es más, al compartir con Chomsky la idea de que la facultad del lenguaje hace parte de la biología humana, ha hecho que su obra se constituya en un nuevo punto de partida para el diseño de una teoría biológica del desarrollo del lenguaje. Precisamente en el capítulo 9 de su libro, propone cinco premisas generales que sostendrán dicha teoría: (i) La función cognitiva es específica para cada especie; (ii) Las propiedades específicas de la función cognitiva aparecen reproducidas en todos los miembros de la especie; (iii) Los procesos y capacidades cognitivos se diferencian espontáneamente con la maduración; (iv) $\mathrm{Al}$ nacer, el hombre está relativamente inmaduro; ciertos aspectos de su conducta y de su función cognitiva surgen solo durante la infancia y (v) Entre los animales, ciertos fenómenos sociales sobrevienen por adaptación espontánea de la conducta del individuo en desarrollo a la conducta de los otros animales que le rodean (pp. 413-415 en la versión española). 
Una nueva lectura del texto de Lenneberg (1967), en diálogo con las propuestas teóricas posteriores, sugiere la posibilidad de consolidar un camino integral sugerido por Bickerton (Calvin \& Bickerton 2001, 16-17): Pero obviamente, una vez que ha quedado establecido que el lenguaje es algo biológicamente determinado, el siguiente paso que alguien deberá dar es tratar de averiguar exactamente cómo evolucionó. Y una vez que ha quedado establecido que el lenguaje hunde sus raíces en la estructura del cerebro, lo inmediato es ir a buscar su asentamiento en ese lugar. Estas tres cosas -lenguaje, evolución y cerebro-, en mi opinión, están interrelacionadas. En realidad, no es posible estudiar ninguna de ellas sin estudiar las demás.

En ese orden de ideas, se puede identificar, siguiendo lo formulado por François (en prensa) la correlación existente entre los estudios sobre la evolución de la facultad del lenguaje y los desarrollos de la gramática generativa, desde los aportes de Chomsky (1965) y Lenneberg (1967) hasta la propuesta de Deacon (2012), una lectura intertextual y programática:

\begin{tabular}{|c|c|c|c|}
\hline & $\begin{array}{l}\text { La facultad del } \\
\text { lenguaje y su } \\
\text { evolución }\end{array}$ & $\begin{array}{l}\text { Teorías de la } \\
\text { gramática }\end{array}$ & $\begin{array}{c}\text { Los tres debates } \\
\text { mayores }\end{array}$ \\
\hline 1965 & & $\begin{array}{l}\text { N. Chomsky, } \\
\text { Aspects of the } \\
\text { Theory } \\
\text { of Language }\end{array}$ & \multirow{3}{*}{ Desarrollos previos } \\
\hline 1967 & $\begin{array}{l}\text { E. Lenneberg, } \\
\text { Biological } \\
\text { Foundations of } \\
\text { Language }\end{array}$ & & \\
\hline 1981 & & $\begin{array}{c}\text { N. Chomsky, } \\
\text { Lectures on } \\
\text { Government and } \\
\text { Binding }\end{array}$ & \\
\hline 1990 & $\begin{array}{l}\text { S. Pinker \& P. } \\
\text { Bloom, } \\
\text { 'Natural language } \\
\text { and natural } \\
\text { selection' } \\
\text { + debate } \\
\text { D. Bickerton, } \\
\text { Language and } \\
\text { Species }\end{array}$ & & \multirow[t]{2}{*}{ Debate $1990-1991$} \\
\hline 1991 & $\begin{array}{l}\text { M. Corballis } \\
\text { The lopsided ape }\end{array}$ & & \\
\hline 1994 & $\begin{array}{l}\text { S. Pinker, } \\
\text { The Language } \\
\text { Instinct }\end{array}$ & & \multirow{4}{*}{$\begin{array}{l}\text { Desarrollos } \\
\text { intermedios }\end{array}$} \\
\hline 1995 & & $\begin{array}{l}\text { N. Chomsky, } \\
\text { The Minimalist } \\
\text { Program }\end{array}$ & \\
\hline 1997 & $\begin{array}{l}\text { T. Deacon, } \\
\text { The Symbolic } \\
\text { Species }\end{array}$ & & \\
\hline 2000 & $\begin{array}{l}\text { L. Jenkins, } \\
\text { Biolinguistics }\end{array}$ & & \\
\hline 2002 & $\begin{array}{l}\text { M. Hauser, N. } \\
\text { Chomsky \& W.T. } \\
\text { Fitch (HCF) }\end{array}$ & $\begin{array}{l}\text { R. Jackendoff, } \\
\text { Foundations of } \\
\text { Language }\end{array}$ & \multirow[b]{2}{*}{ Debate 2002-2005 } \\
\hline 2005 & $\begin{array}{l}\text { S. Pinker \& R. } \\
\text { Jackendoff (P\&J) } \\
\text { W.T. Fitch, N. } \\
\text { Chomsky \& M. } \\
\text { Hauser (FCH) } \\
\text { R. Jackendoff \& S. } \\
\text { Pinker (J\&P) }\end{array}$ & $\begin{array}{l}\text { P. Culicover \& R. } \\
\text { Jackendoff, } \\
\text { Simple Syntax }\end{array}$ & \\
\hline 2007 & $\begin{array}{l}\text { C. Boeckx \& K. } \\
\text { Grohmann } \\
\text { Biolinguistics } 1\end{array}$ & & \\
\hline 2010 & $\begin{array}{c}\text { T. Deacon, } \\
\text { "On the Human - } \\
\text { Rethinking the } \\
\text { natural } \\
\text { selection of human } \\
\text { language " + debate }\end{array}$ & & \multirow[t]{2}{*}{$\begin{array}{l}\text { Debate } 2010 \text { y su } \\
\text { eco en } 2012\end{array}$} \\
\hline 2012 & $\begin{array}{l}\text { Th. Schilhab, F. } \\
\text { Stjernfelt \& T } \\
\text { Deacon } \\
\text { (eds.), The Symbolic } \\
\text { Species Evolved }\end{array}$ & & \\
\hline
\end{tabular}

En 1980 el profesor Jaime Bernal publica en Thesaurus (Tomo XXXV, número 3, pp. 603604) la reseña del libro de Lenneberg en su traducción española. Más que una reseña es un breve opúsculo que registra la aparición de una novedad bibliográfica y pasa a convertirse, como otros tantos, en un texto más de referencia de las asignaturas de lingüística en los programas de licenciatura en lenguas. No se ha establecido aún en Colombia, un diálogo con la obra de Lenneberg.

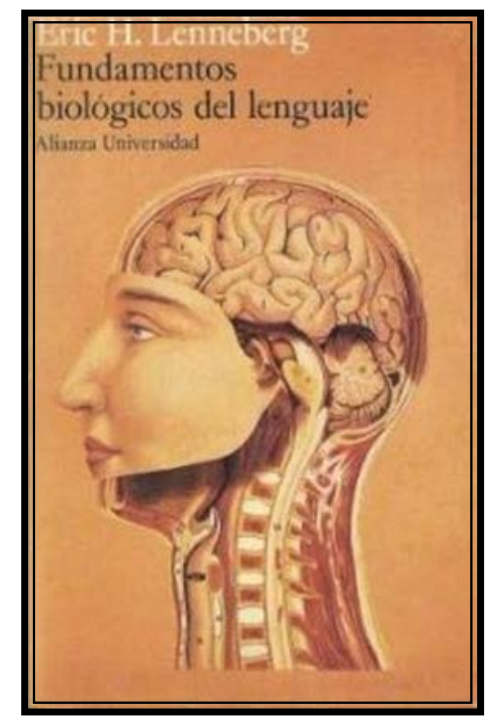

Figura 3. Portada de la edición española de 1975

Entornos se une a dos iniciativas editoriales que le rinden homenaje al profesor Eric Lenneberg. La primera iniciativa es el número especial de la revista Biolinguistics, Volumen 11 (2017) a cargo del editor invitado Patrick C. Trettenbrein. Cada una de las contribuciones propone una nueva lectura de FBL y ofrece datos históricos valiosos para la comprensión del pensamiento de Lenneberg. Los participantes son profesores activos en el ámbito de la biolingüística.

La segunda iniciativa es el libro homenaje que prepara el programa de lengua castellana de la Universidad Surcolombiana y que recoge contribuciones de profesores de Brasil, China, Colombia, España, Francia. El libro consta de tres partes: (i) semblanza biobibliográfica de Lenneberg, (ii) contribuciones teóricas y (iii) traducciones. Se proyecta la publicación del libro para mediados de 2018. 
Como complemento de esta breve semblanza, ofrecemos la traducción del artículo de Bolhuis JJ, Tattersall I, Chomsky N, Berwick RC (2015) Language: UG or Not to Be, That Is the Question.

\section{Referencias bibliográficas}

Lenneberg, E. (1967). Biological Foundations of Language. New York: Wiley.

Lenneberg, E. (1975). Fundamentos biológicos del lenguaje. Madrid: Alianza editorial. Versión española de Natividad Sánchez Sáinz-Trápaga \& Antonio Montesinos.

Chomsky, N. (2017). 50 Years Later: A Conversation about the Biological Study of Language with Noam Chomsky. An interview with Patrick Trettenbrein. Biolinguistics Vol. 11, http://www.biolinguistics.eu/index.php/ biolinguistics/article/view/559/387

Calvin, W. \& Bickerton, D. (2001). Lingua ex Machina. La conciliación de las teorías de Darwin y Chomsky sobre el cerebro humano. Barcelona: Gedisa.

François, J. (en preparación). De la généalogie des langues à la génétique du langage. Une documentation interdisciplinaire raisonnée. Peeters Publisher, Louvain, Bibliothèque de la SLP. 